\title{
Percepción del alumnado sobre la gamificación en la educación superior
}

Nuria Cuevas Monzonís - Universidad Internacional de Valencia

Andrea Cívico Ariza - Universidad Internacional de Valencia

Vicente Gabarda Méndez - Universitat de València

Ernesto Colomo Magaña - Universidad de Málaga
0000-0001-9366-3038

0000-0003-3094-5841

(D) 0000-0001-6159-5173

0000-0002-3527-7937

Recepción: 24.02.2021 | Aceptado: 01.03.2021

Correspondencia a través de ORCID: Vicente Gabarda Méndez

0000-0001-6159-5173

Citar: Cuevas Monzonís, N, Cívico Ariza, A, Gabarda Méndez, V y Colomo Magaña, E (2021). Percepción del alumnado sobre la gamificación en la educación superior. REIDOCREA, 10(16), 1-12.

Resumen: Los procesos formativos se caracterizan por la multiplicidad de planteamientos metodológicos en los que pueden fundamentarse. En este sentido, la gamificación constituye una herramienta de potencial considerable en el diseño e implementación de acciones formativas. Bajo esta perspectiva, el presente artículo pretende conocer la valoración de los estudiantes del Grado en Educación Primaria sobre la utilidad de la gamificación como recurso para la enseñanza y el aprendizaje, tras una experiencia de carácter gamificado. Asimismo, se pretendía identificar posibles diferencias en función del sexo y la edad. La muestra, compuesta por 83 estudiantes ha valorado el recurso mediante de un instrumento ad hoc para la investigación. El instrumento, al margen de recoger información de carácter sociodemográfico, estaba dividido en tres dimensiones básicas para la valoración de la metodología: comunicativa, instrumental y pedagógica. Los resultados muestran que los estudiantes perciben la metodología como útil en sus tres dimensiones (siendo la comunicativa y la pedagógica las que obtienen puntuaciones medias más altas), habiendo una correlación positiva entre todas ellas. La metodología se percibe como especialmente relevante para el desarrollo de la expresión escrita, el aprendizaje significativo, el fomento de la retroalimentación, la promoción del trabajo en grupo o la presentación de contenidos complejos mediante formatos más atractivos. Asimismo, no se han detectado diferencias significativas en función del sexo, pero sí de la edad, vinculándose mayores valoraciones del recurso con una menor edad de los participantes.

Palabra clave: Juego educativo

Student's perception about gamification in higher education

Abstract: Training processes are characterized by the multiplicity of methodological approaches on which they can be based. In this sense, gamification constitutes a tool with an important potential in the design and implementation of formative actions. In this perspective, the present article seeks to know the assessment of the Degree in Primary Education's students about the usefulness of gamification as a resource for teaching and learning, after a gamified experience. It was also intended to identify possible gender and age differences. The sample, composed by 83 students, assessed the resource using an ad hoc instrument for this research. The instrument collected socio-demographic information and was divided into three basic dimensions for the methodology's evaluation: communicative, instrumental and pedagogical. The results show that the students perceive as useful the methodology in its three dimensions (especially the communicative and pedagogical ones), with a positive correlation between all of them. The methodology is perceived as particularly relevant for the development of written expression, meaningful learning, the feedback promotion, the promotion of group work or to present complex content in more attractive formats. In addition, no significant differences have been detected in terms of sex, but in terms of age, with higher assessments of the resource being linked to a younger age of the participants.

Keyword: Educational games

\section{Introducción}

A pesar de que la gamificación pueda concebirse como un fenómeno reciente, e incluso reconocido como tendencia de próxima implantación por parte de los organismos internacionales a través del Informe Horizon, la utilización del juego como metodología educativa cuenta con una tradición considerable.

Sin embargo, la integración de las Tecnologías de la Información y la Comunicación en los procesos de enseñanza y aprendizaje ha contribuido a un renacimiento de la 
consideración del juego como un recurso enriquecedor de las acciones formativas.

Desde una aproximación conceptual, la gamificación tiene su origen en el vocablo inglés gaming, que hace referencia al propio proceso de juego o los juegos de simulación. De este modo, su aplicación en el diseño e implementación de procesos de enseñanza y aprendizaje se vinculan con el game-based learning (GBL) o aprendizaje basado en juegos (Contreras, 2016). Implica, en ocasiones, la utilización de medios electrónicos (videojuegos, tabletas u otros dispositivos) que aportan, a la idiosincrasia del juego, una dimensión audiovisual que se aproxima a la realidad digital actual.

En este sentido, la gamificación entendida como metodología didáctica, se va haciendo presente de manera rápida en los contextos educativos (Sierra \& Fernández, 2019), sirviendo como elemento atrayente para los estudiantes de las diferentes etapas, aproximándonos a sus intereses y motivaciones, y generando una influencia cultural y social notable (Dorado \& Gewerzc, 2017). Igualmente, el abordaje del desarrollo de las competencias desde una perspectiva más lúdica y vivencial supone una redefinición de los procesos formativos y la experiencia de aprendizaje discente, a la vez que contribuye a la creación de nuevos escenarios donde poder diseñarlos e implementarlos. Estas cuestiones han permitido consolidar la gamificación como una metodología habitual para el desarrollo de acciones formativas en las diferentes etapas.

Cabe destacar que el diseño de actividades vinculadas a esta metodología responde a un proceso planificado e intencional (Pariente, 2016). Así, al margen de la integración de elementos básicos inherentes a la propia metodología como la competición, la cooperación o la exploración, su implementación queda contextualizada curricularmente y es coherente con la propia finalidad del aprendizaje. Esto implica que su incorporación no se limita únicamente a un sistema de recompensas puntuales aleatorias (Sánchez, Young \& Journeau-Sion, 2017), algo que desvirtuaría la naturaleza de la gamificación como proceso planificado y orientado a la consecución de objetivos específicos.

También se hace preciso considerar su potencial como recurso al servicio de los procesos de enseñanza y aprendizaje, siendo reseñable en cuestiones diversas. Por un lado, es precisamente su diseño planificado y la identificación de metas el que permite encontrar los puntos básicos de consecución de logros, los cuales tienen asociados reconocimientos y recompensas, contribuyendo así tanto a estimular la curiosidad y mantener la motivación de los estudiantes (Armstrong \& Landers, 2018; Kostenius, Hallberg \& Lindqvist, 2018), como a la mejora del rendimiento de los mismos en términos de calificación (Domínguez et al, 2013). Asimismo, su organización y desarrollo se vincula a un rol activo y participativo del discente (Arnold, 2014), convirtiéndole en eje fundamental de la experiencia y del aprendizaje, lo que favorece un replanteamiento de los roles tradicionales de los agentes. Esta nueva dinámica se caracteriza por un rol docente donde el diseño de la experiencia, la guía y el feedback en el desarrollo y la motivación hacia el logro de metas constituyen los elementos diferenciales (Marín, Vidal, Peirats \& López, 2016). En esta línea, se refuerza la interactividad entre estudiantes y docente y se promueven escenarios de aprendizaje inmersivos donde todos ellos desarrollan tareas explícitas que forman parte de un proceso de construcción conjunto de aprendizaje.

Además, esta participación activa del estudiante, basada en la concentración, el esfuerzo y la motivación (Sánchez, 2015) es inherente al desarrollo de capacidades y destrezas básicas como la autonomía, el trabajo en equipo, la colaboración, las habilidades comunicativas o la competencia digital (Marín-Díaz, 2018).

En esta línea, y tal y como apuntábamos anteriormente, en los últimos tiempos se han 
llevado a cabo experiencias de gamificación en diversos ámbitos y etapas educativas que han puesto de manifiesto el potencial de esta metodología tanto para abordar contenidos como para el desarrollo de competencias de diversa índole. Recogemos, a continuación, los beneficios y ventajas de algunos ejemplos ilustrativos de procesos educativos gamificados desarrollados.

Comenzamos con Gil (2019), quien propone la introducción de experiencias gamificadas con familias, estudiantes y profesores de las etapas de infantil y primaria ya que, tras su implantación, son valoradas de un modo positivo por los diferentes agentes. Por otro lado, Del Moral, Guzmán \& Fernández (2018) utilizan esta metodología para el desarrollo de competencias en el área lingüística, matemática y científica en la etapa de Educación Primaria. Bajo la misma perspectiva de que este recurso tiene un carácter multidisciplinar, podemos encontrar experiencias como la de García, Valls y Gisbert (2019), quienes introducen la gamificación en las asignaturas de Física y Química y Matemáticas en Educación Secundaria Obligatoria. Asimismo, el trabajo de Herrera y Prendes (2019) nos permite observar el potencial de la introducción de experiencias gamificadas en el marco de las asignaturas de Tecnología Industrial y Economía respectivamente, en la etapa de Bachillerato, mientras que Peirats, Marín y Vidal (2017) confían en las posibilidades de esta estrategia metodológica para la atención a la diversidad.

Sin embargo, el interés de este fenómeno se ha ido consolidando también en el ámbito de Educación Superior (Contreras \& Eguia, 2016), siendo especialmente destacable su implementación en los títulos vinculados a la formación docente. Ejemplo de ello, es la experiencia de Agreda, Ortiz, Tallón y López (2019), quienes diseñaron una propuesta basada en la gamificación para la formación del profesorado de las diferentes etapas educativas.

Todas estas consideraciones y experiencias contribuyen a la identificación de una serie de rasgos que fundamentan los beneficios del diseño e implementación de estrategias gamificadas en los procesos formativos en diferentes aspectos:

- En el diseño del propio proceso formativo, pues permite abordar competencias complejas desde una perspectiva lúdica (Oliva, 2016), mientras que su estructuración modular facilita la organización y secuenciación de la acción formativa y, por tanto, del aprendizaje (Gabarda \& Colomo, 2019).

- En su implementación, es clave el cambio de roles tradicional entre docentes y discentes (Sánchez-Mena \& Martí-Parreño, 2016), convirtiendo el protagonismo del alumnado y su participación en uno de los ejes de éxito (Téllez \& Iturriaga, 2014).

- Este cambio de roles y el protagonismo del estudiante, ligado a los principios de recompensa del proceso gamificado, se vinculan directamente con el desarrollo de la motivación intrínseca (Carenys, Moya \& Vila, 2017).

- El proceso gamificado se vincula con el aprendizaje basado en problemas, la creatividad, así como en el aprendizaje experiencial y el aprendizaje significativo (Sánchez-Mena \& MartíParreño, 2016).

- La experiencia gamificada impacta sobre la mejora del rendimiento, la formación y el interés (Barokati, Setyosari, Kuswandi, \& Dwiyogo, 2018).

- La gamificación implica una mejora del compromiso (Faiella \& Ricciardi, 2015), así como un aumento de la autonomía, la autoconfianza y la autoestima del alumnado (Marín, 2015).

- Fomenta el aprendizaje cooperativo, favoreciendo dinámicas de trabajo grupal y mejorando el clima de aula (Hanus \& Fox, 2015).

- El cambio en el rol del docente se materializa en tareas de diseño, guía y retroalimentación, intensificándose esta última tanto en frecuencia como en inmediatez (Kapp, 2012).

En otro orden, investigaciones previas han tratado de aproximarse a la evaluación de la gamificación y a la percepción del alumnado sobre su uso como metodología. En este 
sentido, y desde un punto de vista más instrumental, Torres-Toukoumidis, RamírezMontoya y Romero-Rodríguez (2018) concluían que algunas aplicaciones (Duolingo, Kahoot y Lumosity) cuentan con un mayor potencial para su utilización formativa en base a criterios como la tipología de actores, la motivación para el aprendizaje, la creación y manteamiento de expectativas y el control del usuario. Por su parte, Fernández-Mesa, Olmos-Peñuela y Alegre (2016) concluían que, según la percepción de los estudiantes, la gamificación resultaba una estrategia útil e interesante para la implementación de los procesos de aprendizaje. En la misma línea, Bicen y Kocakoyun (2018), teniendo en cuenta la opinión del alumnado, concluyeron que la experiencia gamificada favoreció la mejora de la motivación, de las áreas donde anteriormente tenían mayores dificultades, así como a un aumento del aprendizaje cooperativo.

Es importante matizar, en este punto, que nuestro estudio se orienta hacia la valoración de la gamificación como recurso para el aprendizaje a través de una experiencia gamificada. Sin embargo, esta experiencia se fundamenta en la utilización de otros recursos (videoblog, redes sociales, publicidad y análisis de portales y webs institucionales) que, por sí mismo, cuentan con un potencial destacable para los procesos de enseñanza y aprendizaje (Santana, Eirín \& Marín, 2017).

En este sentido, nuestro estudio parte del diseño de una experiencia gamificada para la asignatura de Derechos Humanos y Educación en un mundo globalizado. Teniendo en cuenta que la asignatura se dividía en cuatro bloques de contenidos (axiología, multiculturalidad/interculturalidad, inclusión e igualdad de género), la propuesta se construyó en torno a un elemento cultural que permitiera vehicular los diferentes apartados y cuya historia sirviera como hilo conductor respecto al progreso durante la gamificación. La elección fue el cortometraje "El circo de las mariposas" (Weigel, 2009), una ficción sobre resiliencia que permite analizar la diferencia entre comportamientos moralmente reprochables e irreprochables, en función de la relevancia que se le conceda al factor humano y las relaciones positivas entre las personas. Para cada bloque de contenidos, el alumnado tenía unas instrucciones sobre las tareas a realizar, unos recursos preestablecidos para trabajar durante el proceso formativo, así como unos retos y niveles de consecución de logro en función de lo aprendido.

De este modo, en el bloque sobre axiología se trabajará a través de las redes sociales. El cortometraje presenta dos tipos de circos: el primero se construye sobre las deficiencias, mientras que el segundo se edifica en torno a las potencialidades. Este hecho da lugar a reflexionar sobre los comportamientos ético-morales a nivel personal y social de las personas, siendo la formación en valores y la apuesta por aquellos de polaridad positiva la mejor forma de salvaguardar el cumplimiento de los Derechos Humanos. Por esta cuestión, a través de publicaciones, post, elementos compartidos e interacciones (utilizando las herramientas que cada red social pone a disposición del usuario), se pretende que el alumnado debata sobre cuestiones axiológicas (teorías, escuelas de pensamiento, jerarquías de valores, etc.) que les permita tomar conciencia de la importancia de los valores en la esfera de los derechos humanos.

Los contenidos sobre multiculturalidad/interculturalidad utilizarán como recurso el videoblog. Los integrantes del circo de las mariposas pertenecen a diferentes culturas, siendo su unión clave para una convivencia positiva que se manifiesta en su actitud positiva al llevar la ilusión y el entretenimiento a sus espectadores, denotando como la colaboración y una actitud intercultural enriquece el desarrollo personal de los seres humanos. Mediante un trabajo de investigación sobre la convivencia entre las culturas y tomando una cultura minoritaria en su contexto como referencia, el alumnado deberá argumentar sobre las ventajas de las relaciones interculturales, utilizando el videoblog como medio de difusión, interacción y debate entre las aportaciones que realizan el resto 
de compañeros, permitiendo así ampliar el conocimiento sobre las diferentes culturas que conviven en nuestra realidad y la importancia de establecer relaciones positivas entre las mismas.

Para el apartado de inclusión se trabajó respecto al análisis de portales y webs institucionales. La historia de Will refrenda como un contexto inclusivo en el que se promueva la equidad entre las personas, teniendo en cuenta sus diferencias, favorece tanto el desarrollo de sus potencialidades como una adaptación más enriquecedora a la realidad. El reto del alumnado consistía en conocer y analizar las referencias legislativas que determinan la realidad de la inclusión en las distintas esferas de la vida (con especial hincapié en el ámbito educativo), así como las propuestas y proyectos desarrollados por diferentes instituciones para lograr y alcanzar una inclusión real y positiva.

Finalmente, la igualdad de género fue abordada desde la publicidad. En el corto podemos comprobar como Anna sufre humillaciones, maltrato y desigualdades en su vida anterior a unirse al circo de las mariposas cuando se queda embarazada. Teniendo en cuenta que el artículo 1 de los derechos humanos nos iguala a todas las personas por nuestra condición de seres humanos con independencia de la raza, género o cultura, los estudiantes situarán el foco en analizar la situación actual respecto a la igualdad de género a partir de la publicidad. Se les facilitarán anuncios publicitarios recopilados de diversos medios (prensa, radio, televisión, internet, etc.), siendo su labor realizar un análisis pormenorizado de los mismos que les permita tomar conciencia de la situación actual. Para conocer los niveles de consecución o logro, asociados a cada bloque de contenidos, se implementaron diferentes cuestionarios a través de Kahoot, con distintos grados de dificultad, cuyas preguntas abordaban lo trabajado en cada apartado con relación al corto y los recursos utilizados, incluyendo cuestiones fruto de los materiales, intervenciones o creaciones realizadas por los estudiantes del grupo-clase.

\section{Objetivos o hipótesis}

En base a todo lo expuesto anteriormente, el presente trabajo tiene como objetivo principal conocer la percepción de los estudiantes sobre la utilidad de la gamificación como metodología de aprendizaje en base a sus dimensiones comunicativa, pedagógica e instrumental.

Adicionalmente, se pretende comprobar si existen diferencias significativas en las percepciones de los estudiantes según su sexo y su edad, así como analizar la presencia de correlaciones entre las dimensiones que conforman el instrumento.

\section{Métodos}

\section{Enfoque metodológico}

Se plantea un estudio cuantitativo de carácter descriptivo y correlacional. Partiendo de un diseño no experimental y evitando cualquier manipulación sobre las variables de estudio, se recogerá información utilizando cuestiones cerradas formulada de forma estandarizada a la muestra con el propósito de poder cuantificar y tratar de forma estadística las valoraciones aportadas por la misma.

\section{Muestra}

Respecto a la muestra, se ha seleccionado a través de un procedimiento no probabilístico del tipo de conveniencia o causal (Sabariego, 2012), debido a que la población está conformada por alumnado de la asignatura "Derechos Humanos y 
Educación en un mundo globalizado", la cual fue impartida por parte del equipo investigador en el curso 2018-2019. La muestra la componen 83 estudiantes $(\mathrm{N}=83)$ del Grado en Educación Primaria de la Universidad Internacional de Valencia. De los mismos, un total de 61 estudiantes son mujeres (73,5\%) y 22 hombres (26,5\%). Respecto a la edad, la muestra la componen sujetos de entre 24 y 47 años, siendo la media de 32,6 años. Es preciso indicar que, para realizar los correspondientes análisis de los datos, codificamos las edades en tres rangos: 1) 20 a 29;2) 30 a 39; 3) 40 a 49.

\section{Instrumento}

Partiendo del interés por conocer la valoración del alumnado sobre la gamificación como recurso educativo, se ha diseñado un instrumento ad hoc para realizar la investigación. En la primera parte del instrumento se recoge información de carácter sociodemográfico sobre el alumnado, como el sexo (hombre y mujer) o la edad (utilizando los rangos anteriormente descritos). En la segunda parte, se establecen un total de 21 ítems vinculados a 3 dimensiones (comunicativa, instrumental y pedagógica), que nos permitan conocer las fortalezas/debilidades del uso de la gamificación en los procesos de enseñanza-aprendizaje. Su construcción se ha sustentado en el Marco Común de Competencia Digital Docente (2017) y en una selección de propuestas de diferentes investigadores sobre los criterios a considerar para evaluar los recursos didácticos (Morales, Gómez \& García Peñalvo, 2008; Nokelainen, 2006; Pinto, Gómez, Fernández \& Doucet, 2017), cuyo análisis ha permitido incorporar aquellas cuestiones vinculadas al objeto de estudio. Las dimensiones contempladas son la comunicativa (C), donde se analizarán las posibilidades que aporta la gamificación para construir, trasladar y presentar información; la instrumental (I), vinculada con las características que como recurso educativo tiene la gamificación, situando el foco en la funcionalidad de las diferentes herramientas que se integren durante la implementación; y pedagógica $(P)$, contemplando cómo los diferentes aspectos que integran la gamificación influyen en los procesos de enseñanza-aprendizaje. De este modo, el instrumento queda constituido por tres dimensiones con siete ítems cada una (véase tabla 1), cuyas valoraciones, mediante un formato tipo Likert (completamente en desacuerdo, en desacuerdo, ni en desacuerdo ni de acuerdo, de acuerdo y completamente de acuerdo) cuyas puntuaciones oscilarán entre 1 y 5 puntos respectivamente, permitirán conocer la percepción respecto al potencial de la gamificación.

Tabla 1. Dimensiones, ítems del instrumento y codificación

\begin{tabular}{llc}
\hline Dimensión & Ítem & Codificación \\
\hline & Fomenta la expresión oral & C1 \\
& Favorece la expresión escrita & $\mathrm{C} 2$ \\
& Impulsa la capacidad de argumentación & $\mathrm{C} 3$ \\
Comunicativa & Contribuye a difundir y compartir contenidos & $\mathrm{C} 4$ \\
& Mejora la capacidad de comunicación en público & $\mathrm{C} 5$ \\
& Promueve la interacción social & $\mathrm{C} 6$ \\
& Contribuye a la adecuación, claridad y comprensión de la información & $\mathrm{C} 7$ \\
\hline \multirow{5}{*}{ Instrumental } & 11 \\
& Permite presentar en formato multimedia contenidos complejos & 12 \\
& Favorece el desarrollo de la competencia digital & 13 \\
& Fomenta la capacidad atencional & 14 \\
& Favorece la motivación para el aprendizaje & 15 \\
& Es un recurso flexible y adaptable a diferentes contenidos & 16 \\
& Favorece el desarrollo de la creatividad & 17 \\
\hline & Establece grados de dificultad progresivos para el aprendizaje & $\mathrm{P} 1$ \\
& Fomenta la retroalimentación (feedback y evaluación por pares) & $\mathrm{P} 2$ \\
& Contribuye al desarrollo del pensamiento crítico & $\mathrm{P} 3$ \\
& Favorece el aprendizaje significativo & $\mathrm{P} 4$ \\
Pedagógica & Fomenta el aprendizaje autónomo & $\mathrm{P} 5$ \\
& Promueve el trabajo en grupo & $\mathrm{P} 6$ \\
& Ayuda al desarrollo de las competencias & $\mathrm{P} 7$ \\
\hline
\end{tabular}


El instrumento responde tanto al criterio de fiabilidad, obteniéndose un índice de Alfa de Cronbach bueno con un valor de 0,854 , como al de validez mediante juicio de expertos. Este cometido ha recaído en profesores especialistas en Tecnologías de la Información y la Comunicación vinculados a la Universidad Internacional de Valencia y cuyas líneas de investigación se vinculaban con la tecnología educativa y la innovación. En este sentido, su labor ha sido evaluar los criterios de validez, adecuación, inteligibilidad y el principio de exclusividad de los ítems en las correspondientes dimensiones, realizando para ello el análisis descriptivo de la media y el cociente de variación para cada criterio. Utilizando la escala de valoración facilitada a los expertos (Nada adecuado/claro/importante $=0$; algo adecuado/claro/importante $=1$; Bastante adecuado/claro/importante $=2$; muy adecuado/claro/importante $=3$ ), los resultados han reflejado que ninguno de los ítems se situaba por debajo de los límites fijados para revisar o eliminar los mismos (medias inferiores a 1,99 y cocientes de variación superiores al $31 \%$ ), por los que se han mantenido para la realización de la investigación.

\section{Procedimiento y análisis de datos}

Para la recogida de la información, tras la conclusión de la materia, se administró el instrumento al alumnado en forma de cuestionario a través de Google Forms (on line), por la flexibilidad y comodidad que ofrece su carácter asíncrono, así como su gratuidad. Tras recolectar toda la información, se ha procedido a realizar el análisis de los datos mediante el paquete estadístico SPSS v. 25. Partiendo de los objetivos estipulados para este estudio, se han desarrollado diversas pruebas: obtención de los estadísticos descriptivos del conjunto de respuestas del instrumento, prueba $\wedge$ de Wilks y ANOVA, para analizar si hay diferencias significativas en función de las variables sexo y edad; prueba de correlaciones, para comprobar si existen relaciones significativas entre las dimensiones del instrumento.

\section{Resultados}

Teniendo en cuenta los objetivos propuestos para la investigación, seguidamente presentamos los principales resultados obtenidos. Comenzando con un análisis global por dimensiones, el alumnado que ha constituido la muestra de estudio ha valorado el potencial comunicativo que tiene la gamificación como recurso educativo, obteniendo una puntuación media de 4,5 sobre 5 . Muy cercana a esta valoración, se sitúa la dimensión pedagógica $(4,48)$ y la instrumental $(4,34)$, estando todas dentro de la percepción de acuerdo. Además, es preciso señalar que todos los ítems del instrumento han situado sus puntuaciones medias por encima de los 4 puntos, oscilando las valoraciones entre de acuerdo y completamente de acuerdo, denotando así el impacto positivo que la gamificación ha tenido para los estudiantes en el desarrollo de la experiencia formativa. Profundizando en el análisis por dimensión, en lo que respecta a la dimensión comunicativa, las puntuaciones varían entre los 4,33 (contribuye a difundir y compartir contenidos) y los 4,75 (favorece la expresión escrita), obteniéndose la dispersión más baja del instrumento en este último ítem y la mayor heterogeneidad en las respuestas vinculadas a su potencial para impulsar la capacidad de argumentación y su contribución a la adecuación, claridad y comprensión de la información $(0,72$ en ambos casos). En lo que respecta a la dimensión instrumental, las puntuaciones se ubican entre los 4,02 puntos (fomenta la capacidad atencional, siendo el ítem con la dispersión más alta del cuestionario) y los 4,57 puntos (favorece el desarrollo de la creatividad). Respecto a la desviación típica, resaltar como existe mayor acuerdo en el alumnado respecto a que las herramientas utilizadas en el desarrollo de la gamificación permiten presentar en formato multimedia contenidos complejos $(0,66)$, como puede realizarse con el videoblog, por ejemplo. Finalizamos con la dimensión pedagógica, cuyos umbrales de puntuaciones han oscilado entre 4,07 (ayuda al desarrollo de 
competencias) y 4,72 puntos (favorece el aprendizaje significativo), coincidiendo la menor puntuación con el menor acuerdo en las respuestas de los estudiantes ( $D T=1,03)$ y el ítem mejor valorado con la mayor homogeneidad en las respuestas de la muestra $(0,59)$.

Tabla 2. Estadísticos descriptivos

\begin{tabular}{|c|c|c|c|c|c|}
\hline Items & $\mathbf{N}$ & Mín. & Máx. & Media & DT \\
\hline C1: Fomenta la expresión oral & 83 & 3 & 5 & 4,58 & 0,65 \\
\hline C2: Favorece la expresión escrita & 83 & 3 & 5 & 4,75 & 0,49 \\
\hline C3: Impulsa la capacidad de argumentación & 83 & 2 & 5 & 4,60 & 0,72 \\
\hline C4: Contribuye a difundir y compartir contenidos & 83 & 2 & 5 & 4,33 & 0,67 \\
\hline C5: Mejora la capacidad de comunicación en público & 83 & 3 & 5 & 4,36 & 0,66 \\
\hline C6: Promueve la interacción social & 83 & 3 & 5 & 4,43 & 0,65 \\
\hline C7: Contribuye a la adecuación, claridad y comprensión de la información & 83 & 2 & 5 & 4,46 & 0,72 \\
\hline |1: Permite presentar en formato multimedia contenidos complejos & 83 & 3 & 5 & 4,60 & 0,66 \\
\hline |2: Favorece el desarrollo de la competencia digital & 83 & 3 & 5 & 4,18 & 0,72 \\
\hline |3: Fomenta la capacidad atencional & 83 & 1 & 5 & 4,02 & 1,17 \\
\hline 14: Favorece la motivación para el aprendizaje & 83 & 1 & 5 & 4,40 & 0,81 \\
\hline I5: Es un recurso flexible y adaptable a diferentes contenidos & 83 & 2 & 5 & 4,40 & 0,76 \\
\hline |6: Favorece el desarrollo de la creatividad & 83 & 3 & 5 & 4,57 & 0,70 \\
\hline 17: Establece grados de dificultad progresivos para el aprendizaje & 83 & 1 & 5 & 4,24 & 1,05 \\
\hline P1: Fomenta la retroalimentación (feedback y evaluación por pares) & 83 & 2 & 5 & 4,65 & 0,67 \\
\hline P2: Contribuye al desarrollo del pensamiento crítico & 83 & 2 & 5 & 4,43 & 0,70 \\
\hline P3: Favorece el aprendizaje significativo & 83 & 2 & 5 & 4,72 & 0,59 \\
\hline P4: Fomenta el aprendizaje autónomo & 83 & 3 & 5 & 4,27 & 0,73 \\
\hline P5: Promueve el trabajo en grupo & 83 & 2 & 5 & 4,61 & 0,73 \\
\hline P6: Ayuda al desarrollo de las competencias & 83 & 1 & 5 & 4,07 & 1,03 \\
\hline P7: Impulsa la autoevaluación & 83 & 3 & 5 & 4,58 & 0,59 \\
\hline
\end{tabular}

Iniciando el análisis pormenorizado por ítems, las puntuaciones mínimas reflejan que en los ítems 13, 14, 17 y P6, al menos un estudiante ha estado completamente en desacuerdo con la correspondiente afirmación respecto a la gamificación. En el caso de las puntuaciones máximas, destacar que todas las afirmaciones han sido valoradas con el mayor nivel de acuerdo por al menos un estudiante.

Siguiendo con el foco en el análisis por ítems, encontramos que "C2: Favorece la expresión escrita" de la dimensión comunicativa y "P1: Fomenta la retroalimentación (feedback y evaluación por pares)" y "P3: Favorece el aprendizaje significativo" de la dimensión pedagógica, han sido los más valorados por la muestra objeto de estudio, obteniendo todos puntuaciones por encima de 4,64 puntos de media. Además, los ítems C2 $(0,49)$ y P3 $(0,59)$ obtienen el mayor grado de homogeneidad en las respuestas de los estudiantes. En el lado opuesto, se sitúan los ítems "I2: Favorece el desarrollo de la competencia digital" e "I3: Fomenta la capacidad atencional" de la dimensión instrumental y "P6: Ayuda al desarrollo de las competencias" de la dimensión pedagógica, como los peor percibidos por parte de la muestra, siendo relevante que dichas puntuaciones están por encima o igual a 4,02 puntos, de forma que incluso los ítems menos valorados se sitúan en una percepción de acuerdo. Hay que destacar que dos de estos ítems, I3 $(1,17)$ y P6 $(1,03)$, manifiestan el mayor grado de variabilidad, junto con el ítem $17(1,05)$.

Tras realizar el análisis de los estadísticos descriptivos, con el estudio también queríamos evidenciar si había diferencias de carácter significativo en las valoraciones otorgadas por los participantes respecto a las variables sexo y edad. Considerando el sexo de los estudiantes, la prueba $\wedge$ de Wilks $(p=0,286)$ confirma que no existen diferencias significativas ( $p \geq 0,05)$. No obstante, aplicamos el análisis de varianza de una vía para conocer si en algunos de los ítems que componen el instrumento si se habían hallado diferencias, ocurriendo tal caso con el ítem "14: Favorece la motivación para el aprendizaje" (véase tabla 3 ), donde los hombres puntúan más alto que las mujeres. 
Tabla 3. Ítems con diferencias significativa en función de la variable sexo

\begin{tabular}{ccccccc}
\hline Ítem & Sexo & $\mathbf{N}$ & Media & Desv. Típica & F & Sig. \\
\hline \multirow{2}{*}{14} & Hombre & 22 & 4,73 & 0,55 & 5,206 & \multirow{2}{*}{025} \\
& Mujer & 61 & 4,28 & 0,86 & \\
\hline
\end{tabular}

A partir de estos resultados, comprobamos como el sexo no es una variable que influya en la percepción sobre el potencial de la gamificación como recurso educativo para la muestra objeto de estudio. Por el contrario, la variable edad si ha reflejado diferencias significativas entre el alumnado $(p \geq 0,05)$ mediante el $\wedge$ de Wilks $(p=0,000)$. En este caso, recogemos todos los ítems contemplados ya que el ANOVA nos traslada diferencias significativas en 17 de los 21 ítems que conforman el instrumento.

Tabla 4. Ítems con diferencias significativas en función de la variable edad

\begin{tabular}{|c|c|c|c|c|c|c|}
\hline İtem & Edad & $\mathbf{N}$ & Media & Desv. Típica & $F$ & Sig. \\
\hline & $20-29$ & 32 & 4,72 & 0,581 & & \\
\hline \multirow[t]{2}{*}{ C1 } & $30-39$ & 34 & 4,85 & 0,359 & 29,201 & 0,000 \\
\hline & $40-49$ & 17 & 3,76 & 0,562 & & \\
\hline \multirow{3}{*}{$\mathrm{C} 2$} & $20-29$ & 32 & 4,81 & 0,471 & & \\
\hline & $30-39$ & 34 & 4,91 & 0,288 & 12,014 & 0,000 \\
\hline & $40-49$ & 17 & 4,29 & 0,588 & & \\
\hline \multirow{3}{*}{ C3 } & $20-29$ & 32 & 4,81 & 0,471 & & \\
\hline & $30-39$ & 34 & 4,91 & 0,288 & 44,914 & 0,000 \\
\hline & $40-49$ & 17 & 3,59 & 0,795 & & \\
\hline \multirow{3}{*}{ C4 } & $20-29$ & 32 & 4,56 & 0,504 & & \\
\hline & $30-39$ & 34 & 4,44 & 0,613 & 15,420 & 0,000 \\
\hline & $40-49$ & 17 & 3,65 & 0,606 & & \\
\hline \multirow{3}{*}{ C5 } & $20-29$ & 32 & 4,53 & 0,567 & & \\
\hline & 30-39 & 34 & 4,41 & 0,701 & 5,152 & 0,008 \\
\hline & $40-49$ & 17 & 3,94 & 0,556 & & \\
\hline \multirow{3}{*}{ C6 } & $20-29$ & 32 & 4,47 & 0,567 & & \\
\hline & $30-39$ & 34 & 4,68 & 0,475 & 10,614 & 0,000 \\
\hline & $40-49$ & 17 & 3,88 & 0,781 & & \\
\hline \multirow{3}{*}{$\mathrm{C} 7$} & $20-29$ & 32 & 4,59 & 0,560 & & \\
\hline & $30-39$ & 34 & 4,74 & 0,448 & 20,389 & 0,000 \\
\hline & $40-49$ & 17 & 3,65 & 0,862 & & \\
\hline \multirow{3}{*}{11} & $20-29$ & 32 & 4,75 & 0,508 & & \\
\hline & $30-39$ & 34 & 4,82 & 0,387 & 18,091 & 0,000 \\
\hline & $40-49$ & 17 & 3,88 & 0,857 & & \\
\hline \multirow{3}{*}{12} & $20-29$ & 32 & 4,38 & 0,554 & & \\
\hline & $30-39$ & 34 & 4,41 & 0,609 & 21,249 & 0,000 \\
\hline & $40-49$ & 17 & 3,35 & 0,606 & & \\
\hline \multirow{3}{*}{13} & $20-29$ & 32 & 4,19 & 1,030 & & \\
\hline & $30-39$ & 34 & 4,41 & 1,104 & 12,039 & 0,000 \\
\hline & $40-49$ & 17 & 2,94 & 0,899 & & \\
\hline \multirow{3}{*}{14} & $20-29$ & 32 & 4,66 & 0,483 & & \\
\hline & $30-39$ & 34 & 4,59 & 0,657 & 17,183 & 0,000 \\
\hline & $40-49$ & 17 & 3,53 & 1,007 & & \\
\hline \multirow{3}{*}{15} & $20-29$ & 32 & 4,53 & 0,671 & & \\
\hline & $30-39$ & 34 & 4,65 & 0,597 & 13,773 & 0,000 \\
\hline & $40-49$ & 17 & 3,65 & 0,786 & & \\
\hline \multirow{3}{*}{16} & $20-29$ & 32 & 4,75 & 0,508 & & \\
\hline & $30-39$ & 34 & 4,88 & 0,327 & 42,222 & 0,000 \\
\hline & $40-49$ & 17 & 3,59 & 0,712 & & \\
\hline \multirow{3}{*}{17} & $20-29$ & 32 & 4,34 & 1,035 & & \\
\hline & $30-39$ & 34 & 4,41 & 1,076 & 2,917 & 0,060 \\
\hline & $40-49$ & 17 & 3,71 & 0,920 & & \\
\hline \multirow{3}{*}{ P1 } & $20-29$ & 32 & 4,66 & 0,701 & & \\
\hline & $30-39$ & 34 & 4,88 & 0,409 & 7,238 & 0,001 \\
\hline & $40-49$ & 17 & 4,18 & 0,809 & & \\
\hline \multirow{3}{*}{ P2 } & $20-29$ & 32 & 4,59 & 0,615 & & \\
\hline & $30-39$ & 34 & 4,53 & 0,662 & 5,983 & 0,004 \\
\hline & $40-49$ & 17 & 3,94 & 0,748 & & \\
\hline \multirow{3}{*}{ P3 } & $20-29$ & 32 & 4,72 & 0,683 & & \\
\hline & $30-39$ & 34 & 4,88 & 0,409 & 3,845 & 0,025 \\
\hline & $40-49$ & 17 & 4,41 & 0,618 & & \\
\hline
\end{tabular}




\begin{tabular}{|c|c|c|c|c|c|c|}
\hline & $20-29$ & 32 & 4,19 & 0,821 & \multirow{3}{*}{0,430} & \multirow{3}{*}{0,652} \\
\hline \multirow[t]{2}{*}{ P4 } & $30-39$ & 34 & 4,35 & 0,734 & & \\
\hline & $40-49$ & 17 & 4,24 & 0,562 & & \\
\hline \multirow{3}{*}{ P5 } & $20-29$ & 32 & 4,59 & 0,875 & \multirow{3}{*}{5,418} & \multirow{3}{*}{0,006} \\
\hline & 30-39 & 34 & 4,85 & 0,500 & & \\
\hline & $40-49$ & 17 & 4,18 & 0,636 & & \\
\hline \multirow{3}{*}{ P6 } & $20-29$ & 32 & 3,97 & 1,204 & \multirow{3}{*}{0,261} & \multirow{3}{*}{0,771} \\
\hline & $30-39$ & 34 & 4,15 & 0,925 & & \\
\hline & $40-49$ & 17 & 4,12 & 0,928 & & \\
\hline \multirow{3}{*}{ P7 } & $20-29$ & 32 & 4,69 & 0,535 & \multirow{3}{*}{1,265} & \multirow{3}{*}{0,288} \\
\hline & $30-39$ & 34 & 4,56 & 0,561 & & \\
\hline & $40-49$ & 17 & 4,41 & 0,712 & & \\
\hline
\end{tabular}

Si observamos los resultados, los estudiantes pertenecientes al rango de mayor edad (40-47 años) valoran menos la gamificación como recurso educativo que los otros dos grupos de menor edad, convirtiéndose en una constante en todos los ítems en los que se reflejan diferencias significativas. Dicha constante llegar a abarcar dimensiones completas, como la comunicativa, donde en todos los ítems se repite este fenómeno. A tenor de estos resultados, consideramos interesante situar el foco en aquellos ítems en los que no se han producido diferencias significativas por ser menor el número de casos. Entre los mismos, destaca el ítem "I7: Establece grados de dificultad progresivos para el aprendizaje" y "P7: Impulsa la autoevaluación", en cuyos casos el grupo de mayor edad volvía a tener una percepción más baja que la de los grupos de menor edad. Sin embargo, en los ítems "P4: Fomentar el aprendizaje autónomo" y "P6: ayuda al desarrollo de competencias", ha sido el grupo de menor edad (20-29 años), los que han mostrado una menor valoración de la gamificación, resaltando que el alumnado más joven, pese a ofrecer una percepción de acuerdo, valora menos el potencial pedagógico de este recurso en dichos aspectos.

Para finalizar, situamos el interés en comprobar si existían relaciones significativas entre las dimensiones que componen el instrumento, realizando para ello un análisis de correlación que presentamos a continuación.

Tabla 5. Correlaciones entre las dimensiones del instrumento

\begin{tabular}{lccc}
\hline & Comunicativa & Instrumental & Pedagógica \\
\hline Comunicativa & & & \\
Instrumental &, $835^{* *}$ & & \\
Pedagógica &, $514^{* *}$ & $0,547^{* *}$ & \\
\hline${ }^{* *}=p \leq .01$ & & & \\
\hline
\end{tabular}

Los resultados de las correlaciones evidencian una relación positiva alta de la dimensión comunicativa con la instrumental y una relación positiva moderada tanto entre la dimensión comunicativa y la instrumental como entre la instrumental y la pedagógica.

De este modo, vemos como la dimensión comunicativa, los aspectos técnicos de las herramientas utilizadas durante la gamificación, así como su potencial para el aprendizaje están interrelacionados atendiendo a la percepción de los estudiantes que han conformado la muestra. Este hecho denota que, en la experiencia desarrollada, las potencialidades de la gamificación como recurso educativo se extiende a todas las dimensiones contempladas para su análisis, reforzando así su aplicación en el contexto de enseñanza-aprendizaje.

\section{Discusión}

Los resultados analizados nos han permitido conocer la percepción de los estudiantes sobre la utilidad de la gamificación como herramienta para el aprendizaje, siendo esta 
muy alta en consonancia con estudios anteriores como los de Bicen y Kocakoyun (2018) y Fernández-Mesa et al. (2016).

De este modo, hemos podido constatar su utilidad para el desarrollo de competencias asociadas a las tres dimensiones planteadas (comunicativa, instrumental y pedagógica), siendo la primera de ellas la que se ve más beneficiada por la utilización de experiencias gamificadas. De este modo, esta metodología es especialmente útil para la expresión oral y escrita y la capacidad de argumentación, en línea de investigaciones anteriores como la de Area y González (2015). Asimismo, es una herramienta percibida como útil para el desarrollo de competencias relacionadas con la dimensión pedagógica, resultando especialmente poderosa para presentar contenidos complejos en formatos atractivos (Oliva, 2016) y para el desarrollo de la creatividad. Igualmente, los estudiantes perciben la utilidad de la metodología en su dimensión instrumental, especialmente por su capacidad para favorecer el aprendizaje significativo (Sánchez-Mena \& MartíParreño, 2016), para fomentar la retroalimentación (Kapp, 2012) y para el trabajo en grupo (Hanus \& Fox, 2015).

Atendiendo a posibles diferencias en función de algunas variables, no se han podido constatar diferencias en la valoración de la herramienta en función del sexo. Estos resultados, concuerdan con planteamientos como los de Maquilón, Mirete, Sánchez y Hernández (2013) quienes no hallaron diferencias en la valoración de herramientas TIC en procesos de formación de profesorado según esta variable. Sin embargo, sí se han podido evidenciar diferencias significativas en función de la edad, habiendo una relación directa entre una mayor edad y una menor percepción de utilidad. Estos resultados concuerdan con los de Cívico, Colomo, Gabarda y Cuevas (2019).

Todas estas cuestiones nos permiten vislumbrar que la gamificación constituye una propuesta metodológica en auge, siendo su mayor potencial la implicación del alumnado y la motivación que se deprende como aspecto clave para el aprendizaje significativo. En esta línea, se postula como necesario seguir proponiendo planes formativos para el profesorado que les permitan conocer y experimentar metodologías activas y vivenciadas en aras a una mayor calidad y eficacia de los procesos formativos.

\section{Referencias}

Agreda, M, Ortiz, AM, Tallón, S y López, J (2019). Escape Rooms: Actividades gamificadas para la formación de profesionales educativos. En E Sánchez, J Ruiz y E Sánchez (Coords.). Innovación y tecnologías en contextos educativos (pp. 399409). Málaga: UMA Editorial.

Area, M y González, CS (2015). De la enseñanza con libros de texto al aprendizaje en espacios online gamificados. Educatio Siglo XXI, 33(3), 15-38.

Armstrong, MB, \& Landers, RN (2018). Gamification of employee training and development. International Journal of Training and Development, 22(2), 162-169.

Arnold, BJ (2014). Gamification in Education, 21(1), 32-39.

Barokati, N, Setyosari, P, Kuswandi, D, \& Dwiyogo, WD (2018). Gamification as a Strategy to Improve Student Learning Motivation: Preparing Student for 21st Century. International Journal of Engineering \& Technology, 7(2.14), 323-325.

Bicen, H, \& Kocakoyun, S (2018). Perceptions of Students for Gamification Approach: Kahoot as a Case Study. International Journal of Emerging Technologies, 13(2), 72-93.
Carenys, J, Moya, S, \& Vila, M (2017). Effectiveness of an accounting videogame in terms of attributes, motivation and learning outcomes. Revista Internacional de Organizaciones, 18, 29-55.

Cívico, A, Colomo, E, Gabarda, V y Cuevas, N (2019). Herramientas tecnológicas y procesos de tutorización: percepción de los tutores sobre su adecuación. En E Sánchez, J Ruiz y E Sánchez (Coords.), Innovación y tecnologías en contextos educativos (pp. 333-342). Málaga: UMA Editorial.

Contreras, RS y Eguia, JL (2016). Gamificación en aulas universitarias. Bellaterra: Institut de la Comunicació, Universitat Autònoma de Barcelona.

Del Moral, ME, Guzmán, AP, \& Fernández, C (2018). Game-Based Learning: Increasing the Logical-Mathematical, Naturalistic, and Linguistic Learning Levels of Primary School Students. Journal of New Approaches in Educational Research, 7(1), 3139.

Domínguez, A, Saenz, J, De Marcos, L, Fernández, L, Pagés, C, \& Martínez, JJ (2013). Gamifying learning experiences: Practical implications and outcomes. Computers \& Education, 63, 380392. 
Dorado, S y Gewerc, A (2017). El profesorado español en la creación de materiales didácticos: Los videojuegos educativos. Digital Education Review, 31, 176-195.

Faiella, F, \& Ricciardi, M (2015). Gamification and learning: a review of issues and research. Journal of e-Learning and Knowledge Society, 11(3), 13-21.

Fernández-Mesa, A, Olmos-Peñuela, J y Alegre, J (2016). Valor pedagógico del repositorio común de conocimientos para cursos de dirección de empresas. @tic. revista d'innovació educativa, 16, 39-47.

Gabarda, V y Colomo, E (2019). Metodologías didácticas para el aprendizaje en línea. ReiDoCrea. Revista de Docencia Creativa, 8(2), 19-36.

Garcia, F, Valls, C y Gisbert, M (2019). Diseño e implementación de un cambio metodológico en el ámbito científico mediante la gamificación y el modelo de las 5E. Edutec, 66, 65-78.

Gil, J (2019). Interconectados apostando por la construcción colectiva del conocimiento. Aprendizaje móvil en educación infantil y primaria. Píxel-BIT, 54, 185-203.

Hanus, MD, \& Fox J (2015), Assessing the effects of gamifcation in the classroom: A longitudinal study on intrinsic motivation, social comparison, satisfaction, effort, and academic performance. Computer \& Education, 80, 152-161.

Herrera, G y Prendes, MP (2019). Implementación y análisis del método de aula invertida: un estudio de caso en Bachillerato. INNOEDUCA. International Journal of Technology and Educational Innovation, 5(1), 24-33.

Instituto Nacional de Tecnologías y Formación del Profesorado (2017). Marco Común de Competencia Digital Docente.

Kapp, K (2012). The gamification of learning and instruction: gamebased methods and strategies for training and education. New York: John Wiley \& Sons.

Kostenius, C, Hallberg, J, \& Lindqvist, AK (2018). Gamification of health education - Schoolchildren's participation in the development of a serious game to promote health and learning. Health Education. 118(4), 354-368.

Maquilón, JJ, Mirete, AB, García, FA y Hernández, F (2013). Valoración de las TIC por los estudiantes universitarios y su relación con los enfoques de aprendizaje. Revista de Investigación Educativa, 31(2), 537-554.

Marín, D, Vidal, MI, Peirats, J y López, M (2016). Gamificación en la evaluación del aprendizaje: ¡Valoración del uso de Kahoot! En REDINE (Ed.), Innovative strategies for Higher Education in Spain (pp. 8-17). Eindhoven, NL: Adaya Press.

Marín-Díaz, V (2018) ¿El poder de la gamificación educativa? The power of educational gamification? EDMETIC, Revista de Educación Mediática y TIC, 7(2), 1-4.

Morales, EM, Gómez, DA y García Peñalvo, FJ (2008). HEODAR: herramienta para la evaluación de objetos didácticos de aprendizaje reutilizables". En: AB Gil, JA Velázquez, FJ García Peñalvo (Coords.), Simposio Internacional de Informática Educativa SIIE 2008 (pp. 181-186). Salamanca: Ediciones de la Universidad de Salamanca.
Nokelainen, P (2006). An empirical assessment of pedagogical usability criteria for digital learning material with elementary school students. Educational Technology \& Society, 9(2), 178197.

Oliva, HA (2016). La gamificación como estrategia metodológica en el contexto educativo universitario. Realidad y Reflexión, 16(44), 29-47.

Parente, D (2016). Gamificación en la educación. En RS Contreras y JL Eguia (Coords.), Gamificación en aulas universitarias (pp. 11-23). Bellaterra: Institut de la Comunicació, Universitat Autònoma de Barcelona.

Peirats, J, Marín, D y Vidal, MI (2019). Bibliometría aplicada a la gamificación como estrategia digital de aprendizaje. Revista De Educación a Distancia, 19(60), 1-26.

Pinto, M, Gómez, C, Fernández, A y Doucet, AV (2017). Evaluareed: desarrollo de una herramienta para la evaluación de calidad de los recursos educativos electrónicos. Investigación bibliotecológica, 31(72), 227-248.

Sabariego, M (2012). El proceso de investigación. En R Bisquerra, (coord.), Metodología de la investigación educativa (pp. 127 163). Madrid: La Muralla.

Sánchez, F. J. (2015). Gamificación. Education in the Knowledge Society, 16(2), 13-15.

Sanchez, E, Young, S, \& Jouneau-Sion, C (2017). Classcraft: From gamification to ludicization of classroom management. Education and Information Technologies, 22(2), 497-513.

Sánchez-Mena, A, \& Martí-Parreño, J (2016). Gamification in Higher Education: Teachers' Drivers and Barriers. 6th Future of Education Conference. Florence (Italy), June 29-July 2 de 2016.

Santana, PJ, Eirín, R y Marín, D (2017). Análisis y evaluación de portales institucionales en España. Los casos de Canarias, Galicia y Valencia. RELATEC, 16(2), 29-48.

Sierra, M y Fernández-Sánchez, M (2019). Gamificando el aula universitaria. Análisis de una experiencia de escape room en educación superior. REXE, 18(36), 105-115.

Téllez, D y Iturriaga, D (2014). Videojuegos y aprendizaje de la historia: la saga assasin's creed. Contextos Educativos. Revista de Educación, 17, 145-155.

Torres-Toukoumidis, Á, Ramírez-Montoya, MS y RomeroRodríguez, LM (2018). Valoración y evaluación de los Aprendizajes Basados en Juegos (GBL) en contextos elearning. Education in the Knowledge Society, 19(4), 109-128.

Weigel, J (Director and Scriptwriter) Weigel, R (Scriptwriter). (2009). The Butterfly Circus. [DVD]. Estados Unidos: Joshua Weigel, Rebekah Weigel, Angie Alvarez. 